\title{
Electrochemical Behaviour of Cobalt Complexes with 2,4-Bis(Trimethylsilylethynyl)Thiophene
}

\author{
M.L. Marcos, ${ }^{a}$ M.C. Moreno, ${ }^{b}$ A. Arnanz, ${ }^{b}$ S. Delgado, ${ }^{b}$ J. González Velasco ${ }^{a, *}$ \\ ${ }^{a)}$ Departamento de Química, ${ }^{b)}$ Departamento de Química Inorgánica. \\ Universidad Autónoma de Madrid. 28049 Madrid. España
}

Received 2 April 2004; accepted in revised form 8 July 2004

\begin{abstract}
The electrochemical behaviour of five different complexes of cobalt with 2,4bis(trimethylsilylethynyl)thiophene was studied, concluding that the presence of aromatic ligands gives rise to electronic communication effects that allow to define them as molecular wires. The stability of the compounds and of the products of oxidative and reductive charge transfers increases at a temperature of $-30{ }^{\circ} \mathrm{C}$.
\end{abstract}

Keywords: cobalt complexes, electronic communication, molecular wires.

\section{Introducción}

Los metales de transición forman complejos con ligandos alquinos cuya química ha centrado el interés de muchos autores [1,2]. Este tipo de compuestos puede presentar aplicaciones como elementos ópticos no lineales, como precursores en la generación de electroluminiscencia, o bien en forma de los denominados "hilos moleculares" [1-5]. Ligandos del tipo alquinilo o poliino, que pueden actuar como puentes, se ha demostrado que resultan eficientes al ofrecer orbitales moleculares que permiten el flujo electrónico, así como la transmisión de efectos electrónicos entre diversos centros redox activos que puedan formar parte del compuesto[6-15]. Se ha comprobado que un cambio en los centros metálicos, o

\footnotetext{
* Corresponding author. E-mail address: jaime.gonzalez@uam.es
} 
en los ligandos alquinos, o en ambos, produce modificaciones en las propiedades electrónicas de los complejos correspondientes $[8,14,15]$. La presencia en estos compuestos de comunicación electrónica debido a su comportamiento como "hilos moleculares," se pone de manifiesto a través de la respuesta electroquímica a que dan lugar los grupos redox electroactivos existentes [1621]. Se ha podido constatar este tipo de comunicación electrónica entre los centros metálicos que forman parte del complejo en algunos compuestos con dos centros de Co [22-25].

Entre los efectos electrónicos que pueden estudiarse, uno de ellos es el que resulta de la participación de los centros metálicos en la deslocalización $\pi$. Si, por aplicación de un determinado potencial, se induce una transferencia electrónica desde uno de los centros metálicos hacia el electrodo eso crea una diferencia de potencial a través de todo el sistema conjugado que actúa como "hilo electrónico" (macroorbital $\pi$ ), que da lugar a un desplazamiento electrónico a través de toda la cadena, con el resultado de que la falta de electrón queda repartida a través de todo el sistema, de tal manera que ambos centros metálicos resultan parcialmente oxidados, aunque la transferencia electrónica se haya producido en un centro metálico concreto. Se habla entonces de un catión deslocalizado. También puede resultar un catión de valencia mixta. Tanto en un caso como en otro, la cadena conjugada actúa como un factor estabilizador del producto de la transferencia de carga.

En recientes estudios hemos puesto de manifiesto que complejos que contienen unidades $\mathrm{Co}_{2}(\mathrm{CO})_{6}$ ó $\quad \mathrm{Co}_{2}(\mathrm{CO})_{4}(\mathrm{~L}-\mathrm{L}) \quad[\mathrm{L}-\mathrm{L}=$ 1,2-bis(difenilfosfino)metano (dppm), 1,2-bis(difenilfosfino)amina (dppa) y 1,2-bis(dimetilfosfino)metano $($ dmpm) ] unidas a través de 1,4-bis(trimetilsilil)butadiino, 1,3,5,tris(trimetilsililetinil)benceno ó 2,5-bis(trimetilsililetinil)tiofeno presentan comunicación entre los centros metálicos [7-9].

Con el fin de demostrar este tipo de efectos, en este trabajo se han preparado complejos que contienen unidades $\mathrm{Co}_{2}(\mathrm{CO})_{6}$ ó $\mathrm{Co}_{2}(\mathrm{CO})_{4}(\mathrm{~L}-\mathrm{L})$ [L-L $=1,2$ - 
bis(difenilfosfino)metano (dppm)] unidas a través del ligando 2,4bis(trimetilsililetinil)tiofeno.

Se obtuvieron de este modo los compuestos 1-6, que aparecen en el Esquema 1, y cuyo comportamiento electroquímico, estudiado utilizando las voltametrías cíclica y de onda cuadrada permitió demostrar la presencia de comunicación electrónica entre los centros metálicos.



\section{Experimental}

El estudio electroquímico de los compuestos se ha llevado a cabo en un electrolito soporte formado por una disolución $0.15 \mathrm{M}$ en hexafluorofosfato de tetrabutilamonio en $\mathrm{CH}_{2} \mathrm{Cl}_{2}$. El $\mathrm{CH}_{2} \mathrm{Cl}_{2}$ se secó sobre $\mathrm{CaH}_{2}$ y se destiló bajo Ar. Los compuestos 1-6 fueron preparados y caracterizados utilizando las técnicas habituales [9]. 
Las técnicas electroquímicas empleadas fueron voltametría cíclica (CV) y voltametría de onda cuadrada (SWV). La célula de trabajo era una célula de tres electrodos. Como electrodos de trabajo se emplearon un hilo de Pt policristalino y un electrodo de carbono vítreo. Como contraelectrodo se empleó una malla de Pt y como electrodo de referencia se usó un electrodo de cuasirreferencia de Ag. Los potenciales están referidos al sistema $\mathrm{Fc}^{*+} / \mathrm{Fc}^{*}\left(\mathrm{Fc}^{*}=\right.$ decametilferroceno), añadido como referencia interna tras una corta serie de medidas, teniendo en cuenta que el $\mathrm{E}_{1 / 2}$ del par ferroceno/ferricinio está a $+0.55 \mathrm{~V}$ vs. $\mathrm{Fc}^{*+} / \mathrm{Fc}^{*}$ en una disolución de $\mathrm{CH}_{2} \mathrm{Cl}_{2}$.

\section{Resultados y discusión}

En un disolvente tal como $\mathrm{CH}_{2} \mathrm{Cl}_{2}$, tanto la oxidación como la reducción de $\mathbf{1}$ conducen a la formación de substancias que se descomponen químicamente en fase homogénea a $25^{\circ} \mathrm{C}$ (mecanismos EC). Los voltamogramas registrados a una velocidad de barrido $v=100 \mathrm{mV} / \mathrm{s}$, muestran una onda de oxidación (Fig. 1), o de reducción (Fig. 2) que no da lugar a las correspondientes ondas de reducción y oxidación debido a la desaparición de las especies electroactivas por reacción química homogénea. La reacción química de descomposición de los productos de las transferencias de carga es altamente dependiente de la temperatura (por lo tanto se caracteriza por un bajo valor de la energía de activación, como es de esperar de cualquier reacción de ruptura de un enlace previamente desestabilizado por la transferencia de carga). Ello se manifiesta porque los voltamogramas registrados a $-30{ }^{\circ} \mathrm{C}$ (Fig. 3 muestra la onda de reducción del compuesto 1 a $-30{ }^{\circ} \mathrm{C}$ ) se caracterizan por presentar ondas cuasirreversibles, indicando que a dicha temperatura el producto de las transferencias de carga no sufre una reacción química perceptible en el tiempo que tarda en hacerse la inversión del barrido. Igualmente, a $25{ }^{\circ} \mathrm{C}$ y, a $v=5 \mathrm{~V} / \mathrm{s}$, aparecen pequeñas ondas, indicando que el tiempo de descomposición es del orden de centésimas de segundo a esa temperatura. 




Figura 1. Voltamograma correspondiente a la oxidación del compuesto 1 a $25{ }^{\circ} \mathrm{C}$ sobre electrodo de platino; $\mathrm{v}=0.1 \mathrm{Vs}^{-1}$.

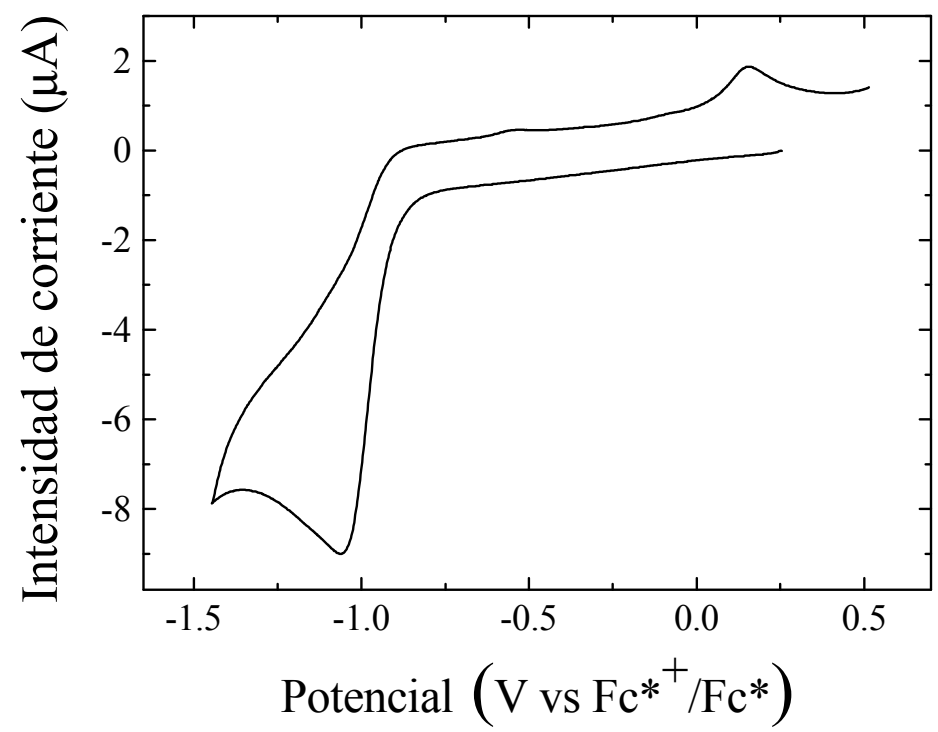

Figura 2. Voltamograma correspondiente a la reducción del compuesto $\mathbf{1}$ a $25{ }^{\circ} \mathrm{C}$ sobre electrodo de platino; $\mathrm{v}=0.1 \mathrm{Vs}^{-1}$.

En los compuestos 2 y 4, la coordinación entre el ligando dppm y el centro $\mathrm{Co}_{2} \mathrm{C}_{2}$ da lugar a un incremento de la densidad electrónica centrada sobre éste, lo que hace que su oxidación se produzca a potenciales menos positivos (facilita su oxidación). La reducción, por el contrario, debido a este incremento en la 
densidad electrónica resulta claramente desfavorecida, lo que se manifiesta en que es necesario aplicar potenciales mucho más negativos (ca. $0.6 \mathrm{~V}$, ver Tabla I) para lograr su reducción. El ligando bidentado también contribuye a aumentar la estabilidad química de los cationes (oxidaciones completamente reversibles a $25^{\circ} \mathrm{C}$ ) y de los aniones (reducciones cuasirreversibles a $25{ }^{\circ} \mathrm{C}$ y completamente reversibles a $\mathrm{T}=-30^{\circ} \mathrm{C}$, ver Fig. 4).

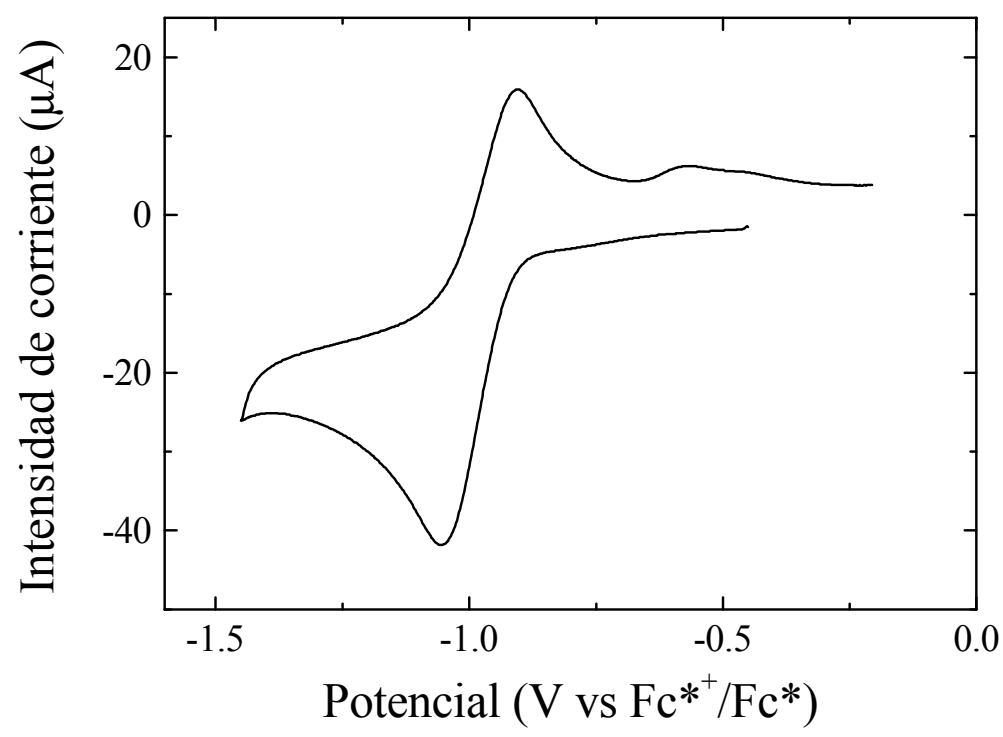

Figura 3. Voltamograma correspondiente a la reducción del compuesto 1 sobre un electrodo de carbono a $-30{ }^{\circ} \mathrm{C} ; \mathrm{v}=0.1 \mathrm{Vs}^{-1}$.

Tabla I. Potenciales de reducción y oxidación de los compuestos 1-2 y 4-6 en disolución de $\mathrm{CH}_{2} \mathrm{Cl}_{2}\left(\mathrm{~V}\right.$ vs. $\left.\mathrm{Fc}^{*+} / \mathrm{Fc}^{*}\right)$.

\begin{tabular}{ccccc}
\hline & $\mathrm{E}_{1 / 2}(\mathrm{red})$ & $\Delta \mathrm{E}_{1 / 2}(\mathrm{red})$ & $\mathrm{E}_{1 / 2}(\mathrm{ox})$ & $\Delta \mathrm{E}_{1 / 2}(\mathrm{ox})$ \\
\hline $\mathbf{1}$ & $-0.99^{\mathrm{a}}$ & & $1.24^{\mathrm{a}}$ & \\
$\mathbf{2}$ & $-1.56^{\mathrm{a}}$ & & $0.69^{\mathrm{b}}$ & \\
$\mathbf{4}$ & $-1.60^{\mathrm{b}}$ & & $0.68^{\mathrm{b}}$ & \\
$\mathbf{5}$ & $-1.00^{\mathrm{a}} ;-1.08^{\mathrm{a}}$ & 0.08 & $1.21^{\mathrm{b}} ; 1.29^{\mathrm{b}}$ & 0.08 \\
$\mathbf{6}$ & $-1.58^{\mathrm{a}} ;-1.72^{\mathrm{a}}$ & 0.14 & $0.65^{\mathrm{b}} ; 0.77^{\mathrm{b}}$ & 0.12 \\
\hline
\end{tabular}

${ }^{\mathbf{a}}$ Medidas a $-30{ }^{\circ} \mathrm{C} .{ }^{\mathbf{b}}$ Medidas a $25{ }^{\circ} \mathrm{C}$ y a $-30{ }^{\circ} \mathrm{C}$. 


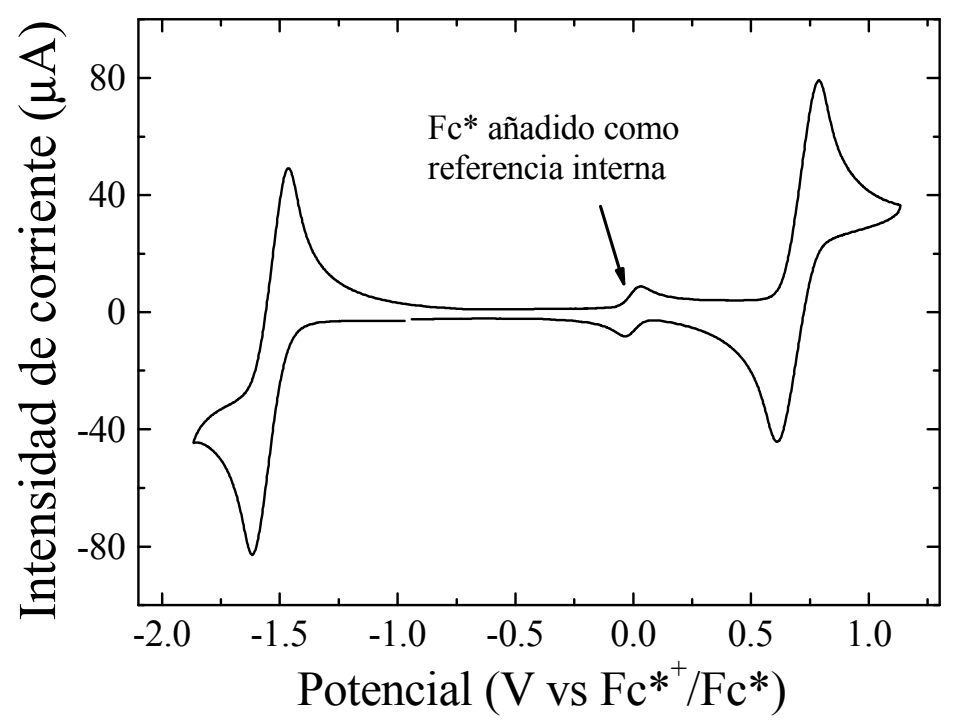

Figura 4. Voltamograma correspondiente a la reducción y la oxidación del compuesto 2 sobre electrodo de carbono a $-30{ }^{\circ} \mathrm{C} ; \mathrm{v}=0.05 \mathrm{Vs}^{-1}$.

En los compuestos disustituidos 5 y 6 tienen lugar dos oxidaciones y dos reducciones monoelectrónicas consecutivas. A temperatura ambiente, la reducción de $\mathbf{5}$ es completamente irreversible, como consecuencia de la inestabilidad del anión resultante que reacciona químicamente, de acuerdo con el esquema EC que se mencionó anteriormente; sin embargo, a $-30{ }^{\circ} \mathrm{C}$ la estabilidad química de las especies reducidas aumenta y es posible apreciar con claridad en el voltamograma cíclico dos ondas diferenciadas con valores de $i_{a} / i_{c}<1$ a 0.1 $\mathrm{Vs}^{-1}$. Además la relación $\mathrm{i}_{\mathrm{a}} / \mathrm{i}_{\mathrm{c}}$ se hace más próxima a 1 al incrementar la velocidad de barrido, tal como es predecible en sistemas electroquímicos de tipo EC (Fig. 5). En el caso del compuesto 6, también presenta dos ondas de reducción a -30 ${ }^{\circ} \mathrm{C}$, la primera de las cuales se comporta como completamente reversible, mientras que la relación entre las corrientes anódica y catódica que se mide en la segunda resulta ser menor de la unidad: $\mathrm{i}_{\mathrm{a}} / \mathrm{i}_{\mathrm{c}}<1$ (Fig. 6). En lo que respecta a los procesos de oxidación, el compuesto 6 da lugar a registros voltamétricos más reversibles, lo que indica que el catión resultante de la transferencia de carga hacia el electrodo es más estable químicamente que lo eran los aniones. 


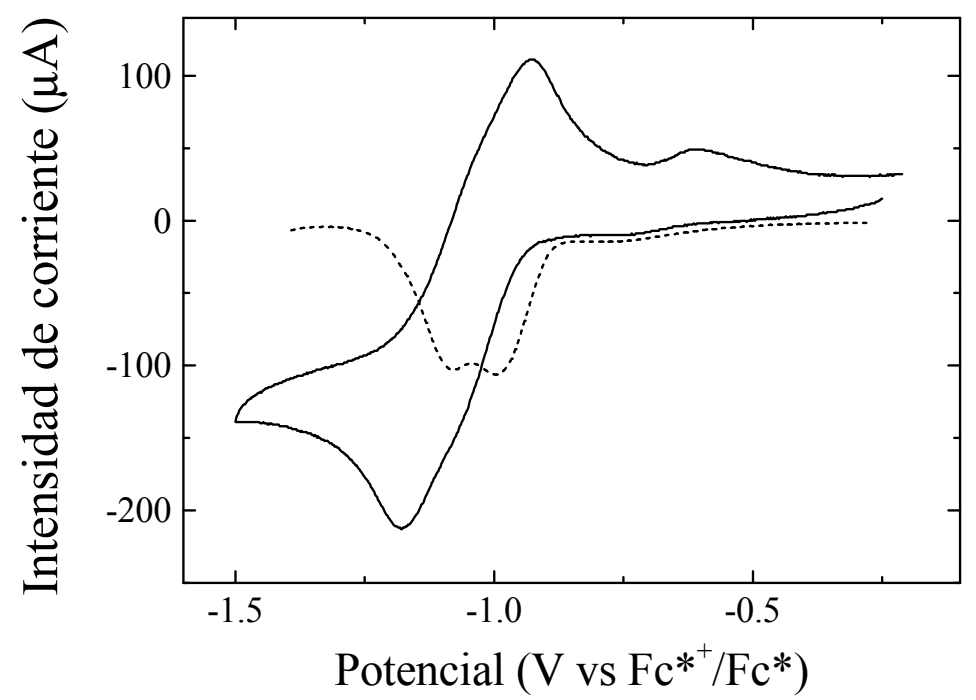

Figura 5. Voltamogramas correspondientes a la reducción del compuesto 5 sobre electrodo de carbono a $-30{ }^{\circ} \mathrm{C}$. Línea continua: voltametría cíclica, $\mathrm{v}=0.5 \mathrm{Vs}^{-1}$; línea discontinua: voltametría de onda cuadrada, $\mathrm{f}=15 \mathrm{~Hz}$.

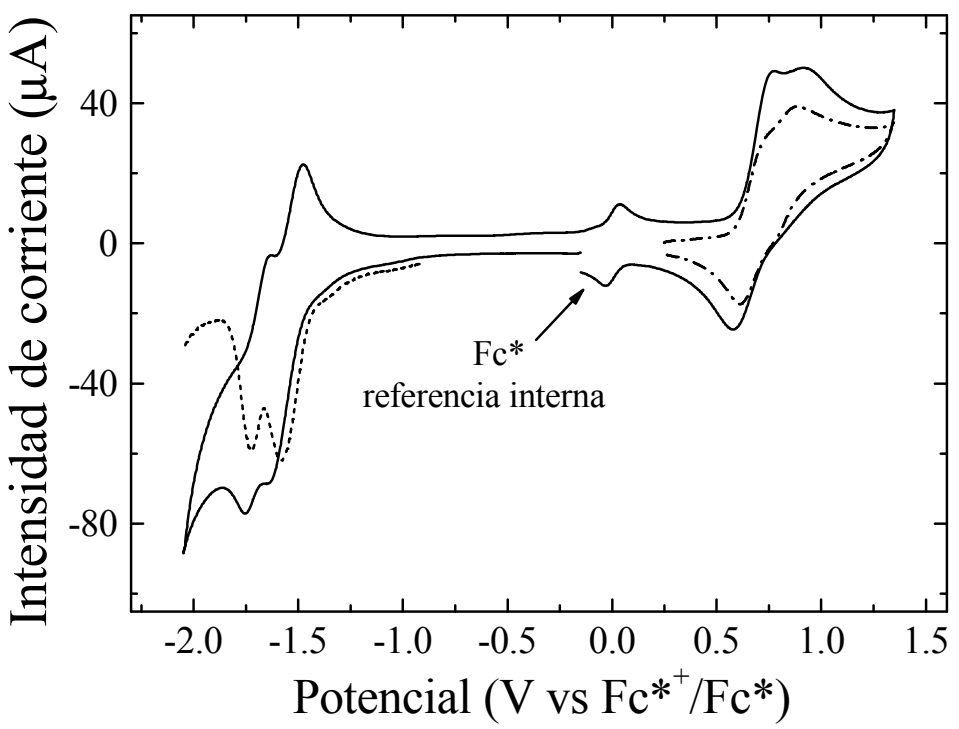

Figura 6. Voltamogramas correspondientes a la reducción y oxidación del compuesto 6. (-) Voltametría cíclica sobre electrodo de carbono a $-30{ }^{\circ} \mathrm{C}, \mathrm{V}=0.1 \mathrm{Vs}^{-1}$; (- -) voltametría de onda cuadrada sobre electrodo de carbono a $-30{ }^{\circ} \mathrm{C}, \mathrm{f}=60 \mathrm{~Hz}$; (- - -) voltametría cíclica sobre electrodo de Pt a $25^{\circ} \mathrm{C}, \mathrm{v}=0.1 \mathrm{Vs}^{-1}$. 
La diferencia entre los potenciales de las ondas consecutivas, $\Delta \mathrm{E}_{1 / 2}$, se suele tomar como una medida de la interacción electrónica que tiene lugar a través de un ligando "puente" entre dos centros redox equivalentes [22]. Para los compuestos 5 y $\quad \mathbf{6}, \Delta \mathrm{E}_{1 / 2}$ es, aproximadamente, 80 y $120-140 \mathrm{mV}$, respectivamente, lo que nos permite calcular el valor de $\mathrm{K}_{\mathrm{C}}$ (constante de comproporción, $\mathrm{RT} \ln \mathrm{K}_{\mathrm{C}}=\mathrm{nF} \Delta \mathrm{E}_{1 / 2}$ ), que resulta ser de 22.5 (5) y de 157 (6). Estos valores indican que entre los dos centros redox existe una comunicación electrónica de baja intensidad que se establece a través del ligando aromático, con lo que los compuestos 5 y $\mathbf{6}$ pertenecen al grupo II en la clasificación de Robin-Day de compuestos de valencia mixta [26].

Las posiciones en que se encuentran las sustituciones en el anillo del tiofeno del ligando puente tienen una clara influencia en la comunicación electrónica observada en los compuestos. De esta manera, hemos observado que en compuestos análogos a 5 y $\mathbf{6}$, pero sustituidos en las posiciones 2 y $5, \Delta \mathrm{E}_{1 / 2}$ (ox) es $200 \mathrm{mV}$ y $290 \mathrm{mV}$, respectivamente [9]. Estos valores son sensiblemente superiores a los de $\mathbf{5}$ y $\mathbf{6}$, e indican una mayor interacción electrónica.

Por otro lado, con otro tipo de ligandos puente, como en $\left[\mathrm{Co}_{2}(\mathrm{CO})_{4} \mathrm{dppm}\right]_{2}$ $\left(\mathrm{Me}_{3} \mathrm{SiC}_{2}(\mathrm{C} \equiv \mathrm{C})_{2} \mathrm{C}_{2} \mathrm{SiMe}_{3}\right) \quad[27] \quad y \quad\left[\mathrm{SiMe}_{3}\left(\mathrm{Co}_{2}(\mathrm{CO})_{4} \mathrm{dppm}\right) \mathrm{C}_{2}\right]_{2}(\mathrm{RC} \equiv \mathrm{C})(1,3,5-$ $\left.\mathrm{C}_{6} \mathrm{H}_{3}\right)\left(\mathrm{R}=\mathrm{SiMe}_{3}[8], \mathrm{H}[27]\right)$ los valores de $\Delta \mathrm{E}_{1 / 2}(\mathrm{ox})$ obtenidos en las mismas condiciones experimentales que las que se han utilizado en la realización del presente estudio fueron de 200, 170 y $150 \mathrm{mV}$, respectivamente, comparables aunque superiores a los $120 \mathrm{mV}$ de 6. Para compuestos con los mismos centros redox que 5, se ha encontrado que $\Delta \mathrm{E}_{1 / 2}(\mathrm{red})$ es de 140 y $180 \mathrm{mV}$ para $\left[\mathrm{SiMe}_{3}\left(\mathrm{Co}_{2}(\mathrm{CO})_{6}\right) \mathrm{C}_{2}\right]_{2}\left(\mathrm{SiMe}_{3} \mathrm{C} \equiv \mathrm{C}\right)\left(1,3,5-\mathrm{C}_{6} \mathrm{H}_{3}\right) \quad$ y $\left[\mathrm{SiMe}_{3}\left(\mathrm{Co}_{2}(\mathrm{CO})_{6}\right) \mathrm{C}_{2}\right]_{3}$ $\left.1,3,5-\mathrm{C}_{6} \mathrm{H}_{3}\right)$, respectivamente [8].

La interacción electrónica es mucho más acusada cuando el ligando puente es butadiino. De esta manera, $\Delta \mathrm{E}_{1 / 2}$ es $450 \mathrm{mV}$ para la oxidación de $\left[\mathrm{Co}_{2}(\mathrm{CO})_{4} \mathrm{dppm}\right]_{2}\left(\mathrm{PhC}_{2} \mathrm{C}_{2} \mathrm{Ph}\right)[28]$, mientras que $\Delta \mathrm{E}_{1 / 2}$ es 400,460 y $480 \mathrm{mV}$ para la reducción en THF de $\left[\mathrm{Co}_{2}(\mathrm{CO})_{6}\right]_{2}\left(\mathrm{RC}_{2} \mathrm{C}_{2} \mathrm{R}\right)$, con $\mathrm{R}=\mathrm{Ph}, \mathrm{CH}_{3} \mathrm{y} \mathrm{SiMe}_{3}$, respectivamente [25]. 


\section{Conclusiones}

Se ha llevado a cabo el estudio electroquímico de 5 diferentes complejos de cobalto con 2,4-bis(trimetilsililetinil)tiofeno. La voltametría cíclica del compuesto 1 pone de manifiesto que la oxidación y la reducción se producen a través de mecanismos $\mathrm{EC}$, en los que la reacción química posterior a la transferencia de carga se hace lenta a temperatura de $-30{ }^{\circ} \mathrm{C}$. En los compuestos 2 y 4, la presencia del ligando donador dppm da lugar a un incremento de la densidad electrónica sobre el centro $\mathrm{Co}_{2} \mathrm{C}_{2}$, lo que permite que la oxidación se produzca a un potencial alrededor de $600 \mathrm{mV}$ menos positivo y la reducción a un potencial $600 \mathrm{mV}$ más negativo que en ausencia de dicho ligando. En los compuestos 5 y $\mathbf{6}$ se demuestra la presencia de interacción electrónica ejercida a través del ligando aromático puente entre dos centros redox equivalentes, por lo que se puede concluir que dichos compuestos actúan como hilos moleculares.

\section{Agradecimientos}

Los autores de este estudio quieren expresar su agradecimiento al Ministerio de Ciencia y Tecnología por la concesión del Proyecto BQU 2002-02522 que hizo posible su realización del mismo.

\section{Comportamiento Electroquímico de Complejos de Cobalto con 2,4 Bis(Trimetilsililetinil)Tiofeno}

\section{Referencias}

1. A. Sappa, A Tiripicchio, P. Braunstein, Chem. Rev. 83 (1983) 203.

2. P.R. Rainthby, M.J. Rosales, Adv. Inorg. Chem. Radiochem. 29 (1985) 169.

3. S. Creager, C.J. Yu, C. Bamdad, S. O'Connor, T. Maclean, E. Lam, Y. Chong, G.T. Olsen, J. Luo, M. Gozon, J.F. Hayyem, J. Am. Chem. Soc. 121 (1999) 1059.

4. P. Stepnicka, R. Gyepes, I. Císarova, Organometallics 18 (1999) 627. 
5. P.I. Dosa, C. Erben, V.S. Iyer, K.P.C. Vollhardt, I.M. Wasser, J. Am. Chem. Soc. 121 (1999) 10430.

6. D.T. McQuade, A.E. Pullen, T.M. Swager, Chem. Rev. 100 (2000) 2537.

7. C. Moreno, J.L. Gómez, R.M. Medina, M.J. Macazaga, A. Arnanz, A. Lough, D.H. Farrar, S. Delgado, J. Organomet. Chem. 579 (1999) 63.

8. C. Moreno, M.L. Marcos, G. Domínguez, A. Arnanz, D.H. Farrar, R. Teeple, A. Lough, J. González-Velasco, S. Delgado, J. Organomet. Chem. 631 (2001) 19.

9. M.L. Marcos, A. Arnanz, C. Moreno, D.H. Farrar, A. Lough, J. Yu, S. Delgado, J. González-Velasco, J. Organomet. Chem., enviada para su publicación.

10. N. Le Narvor, L. Toupet, C. Lapinte, J. Am. Chem. Soc. 117 (1995) 7129.

11. T. Bartik, B. Bartik, R. Dembinski, J.A. Gladysz, Angew. Chem. Int. Ed. Engl. 35 (1996) 414.

12. F. Coat, C. Lapinte, Organometallics 15 (1996) 477.

13. T.S. Jung, J.H. Kim, E.K. Jang, D.H. Kim, Y.-B. Sim, B. Park, S.C. Shin, J. Organomet. Chem 599 (2000) 232.

14. R.D. Adams, B. Qu, Organometallics 19 (2000) 2411.

15. H.J. Jiao, J.A. Gladysz, New. J. Chem. 25 (2001) 551.

16. M.L. Marcos, M.J. Macazaga, R.M. Medina, C. Moreno, J.A. Castro, J.L. Gómez, S. Delgado, J. González-Velasco, Inorg. Chim. Acta 312 (2001) 249.

17. J. Roncali, Chem. Rev. 97 (1997) 173.

18. D.T. McQuade, A.E. Pullen, T.M. Swager, Chem. Rev. 100 (2000) 2537.

19. C.J. Elsevier, J. Reedijk, P.H. Walton, M.D. Ward. J. Chem. Soc., Dalton Trans. (2003) 1869.

20. G.H. Worth, B.H. Robinson, J. Simpson, Appl. Organomet. Chem. 4 (1990) 481.

21. B.H. Robinson. "Chemistry and Reactivity of Metal Cluster Carbonyl Radical Anions in Paramagnetic Organometallic Species in Activation, Selectivity and Catalysis” (Eds.: Kluwer, Dordrecht), 1987. 
22. A.J. Bard, L.R. Faulkner, Electrochemical Methods, Wiley, New York, 1980.

23. H. Yao, M. Sabat, R.N. Grimes, P. Zanello, F. Fabrizi de Biani, Organometallics 22 (2003) 2581.

24. H. Yao, M. Sabat, R.N. Grimes, F. Fabrizi de Biani, P. Zanello, Angew. Chem. Int. Ed. Eng. 42 (2003) 1002.

25. D. Osella, L. Milone, C. Nervi, M. Ravena, Eur. J. Inorg. Chem. (1998) 1473.

26. M.B. Robin, P. Day, Adv. Inorg. Chem. Radiochem. 10 (1967) 247.

27. R.M. Medina, C. Moreno, M.L. Marcos, J.A. Castro, F. Benito, A. Arnanz, S. Delgado, J. González-Velasco, M.J. Macazaga, Inorg. Chim. Acta 35 (2004) 2069.

28. C.J. McAdam, N.W. Duffy, B.H. Robinson, J. Simpson, Organometallics 15 (1996) 3935. 